\title{
Prevalencia y Severidad de Caries Dental en los Niños Beneficiarios del Programa de Salud Oral Asociados a Escuelas de Chile
}

\author{
Prevalence and Severity of Dental Caries in Beneficiary Children \\ in the Oral Health Program Associated with Schools in Chile
}

\begin{abstract}
Gerardo Espinoza-Espinoza, ${ }^{1,2}$ Patricia Pineda1,4; Claudia Atala-Acevedo ${ }^{1,4}$; Patricia Muñoz-Millán ${ }^{1,4}$; Sergio Muñoz ${ }^{2,3}$; Andrea Weits ${ }^{5}$; Bárbara Hernandez ${ }^{5}$; José Castillo ${ }^{5}$ \& Carlos Zaror Ca,4 $^{1,3,4}$
\end{abstract}

\begin{abstract}
ESPINOZA-ESPINOZA, G.; PINEDA, P.; ATALA-ACEVEDO, C.; MUÑOZ-MILLÁN, P. ; MUÑOZ, S.; WEITS, A.; HERNANDEZ, B.; CASTILLO, J. \& ZAROR, C. Prevalencia y severidad de caries dental en los niños beneficiarios del programa de salud oral asociados a escuelas de Chile. Int. J. Odontostomat., 15(1):166-174, 2021.
\end{abstract}

RESUMEN: La caries es la enfermedad crónica más prevalente en niños constituyendo un problema de salud pública a nivel mundial. El objetivo de este estudio fue determinar la prevalencia y severidad de caries en niños y niñas pertenecientes al Programa de Salud Oral asociado a escuelas de la Junta Nacional de Auxilio Escolar y Becas (JUNAEB). Se realizó un estudio de corte trasversal basado en datos del año 2015 del Sistema Informático del Programa de Salud Oral de JUNAEB. Las variables de estudio fueron presencia y severidad de caries (índices ceod y COPD) y las variables de asociación exploratorias fueron zona geográfica, provincias, sexo, tipo de dependencia administrativa del colegio, tipo de enseñanza, sistema de salud, situación de extrema pobreza, tipo de dentición y tipo de atención. La asociación independiente entre las variables se analizó mediante el test de Chi2 y t-test. La muestra quedó constituida por 162.116 individuos, siendo el $50 \%$ mujeres. La población estudiada mostró una prevalencia de $49 \%$ y un índice ceod y COPD de 2,48 y 1,55 respectivamente. La mayor prevalencia (63\%) fue la zona centro sur y la región del Bío-Bío mostró los mayores índices de severidad $(p<0,001)$. Las asociaciones más significativas fueron entre caries y el nivel socioeconómico y zona geográfica $(p<0,001)$. Este estudio evidencia la asociación de la prevalencia/ severidad de caries y el nivel socioeconómico, y la distribución geográfica de la caries; lo cual hace necesario implementar medidas preventivas que compensen la ruralidad o la falta de fluoración del agua en algunas zonas geográficas de pobreza extrema.

PALABRAS CLAVE: caries dental, factores de riesgo, niños, prevaencia, factores socioeconómicos.

\section{INTRODUCCIÓN}

La caries es la enfermedad crónica más prevalente en niños y constituye un problema de salud pública debido a su alta prevalencia, su impacto negativo en la calidad de vida y el alto costo de su tratamiento (Matamala-Santander et al., 2019; Ortiz et al., 2020).
Según la OMS la caries logra afectar entre el $60 \%$ y $90 \%$ de la población escolar y su aparición se asocia con factores socioculturales, económicos, ambientales y del comportamiento (Cerón-Bastidas, 2015; Corrêa-Faria et al., 2016). Otro factor que influye son las barreras para recibir tratamiento dental adecuado:

\footnotetext{
${ }^{1}$ Centro de Investigación en Economía, Epidemiologia y Salud Publica Oral (CIEESPO), Facultad de Odontología, Universidad de La Frontera, Temuco, Chile.

2 Departamento de Salud Pública, Universidad de La Frontera, Temuco, Chile.

${ }^{3}$ Centro de Excelencia CIGES, Facultad de Medicina, Universidad de La Frontera, Temuco, Chile.

${ }^{4}$ Departamento de Odontopediatría y Ortodoncia, Universidad de La Frontera, Temuco, Chile.

5 Junta Nacional de Auxilio Escolar y Becas (JUNAEB), Santiago, Chile.

FINANCIAMIENTO: Este estudio fue financiado por Junta Nacional de Auxilio Escolar y Becas (JUNAEB).
} 
ESPINOZA-ESPINOZA, G.; PINEDA, P.; ATALA-ACEVEDO, C.; MUÑOZ-MILLÁN, P. ; MUÑOZ, S.; WEITS, A.; HERNANDEZ, B.; CASTILLO, J. \& ZAROR, C. Prevalencia y severidad de caries dental en los niños beneficiarios del programa de salud oral asociados a escuelas de Chile. Int. J. Odontostomat., 15(1):166-174, 2021.

barreras de acceso y utilización de servicios odontológicos (Hernández-Vásquez et al., 2016), donde los factores económicos influyen en el acceso a los servicios de salud y la capacidad de los individuos para obtener cobertura (Palomer Roggerone, 2016).

La Encuesta Nacional de Salud 2009-2010, evidenció que en Chile las disparidades de salud bucal existen para muchos grupos, por nivel socioeconómico, educacional, género, edad, etnia y ubicación geográfica (Ministerio de Salud, 2011a). Reportes del Ministerio de Salud (MINSAL), indican que la caries dental se presenta desde los primeros años de vida; a los 2 años la prevalencia de caries es de un $17,5 \%$, de $49,6 \%$ a los 4 años, de $70,4 \%$ a los 6 años y a los 12 años la prevalencia de caries es de 62,5 (Soto et al., 2007; Ministerio de Salud, 2011b). Existe evidencia que la prevalencia y severidad del daño por caries en la población infanto-adolescente están relacionados con inequidades en salud (Lambert et al., 2017), afectando a los más desfavorecidos e incrementando con la edad (Soto et al.; Soto \& Tapia, 2007; Fernández González et al., 2011). Otro factor analizado en los estudios nacionales es la mayor prevalencia que presentan los niños y adolescentes de zonas rurales en comparación con los que viven en zonas urbanas, con una diferencia desde un $18 \%$ a un $38 \%$ (Soto et al.; Giacaman et al., 2015; Cabrera et al., 2015).

El Plan Nacional de Salud Bucal 2018-2030, establece que la salud bucal es una prioridad en el país, siendo parte integral del bienestar de las personas, familias y comunidades. En respuesta a esto, se han formulado e implementado programas derivados de las diferentes políticas y estrategias en salud bucal, con el objetivo de disminuir las inequidades y brechas detectadas en nuestro país (Ministerio de Salud, 2018).

Para hacer frente a estas desigualdades nace en el año 1991, el Programa de Salud Oral de la Junta Nacional de Auxilio Escolar y Becas (JUNAEB) y desde ese entonces a la fecha, se mantiene como el único programa de carácter público y gratuito que otorga atención odontológica integral, sistemática y programada del estudiante de educación parvulario (a) y enseñanza básica, es decir, desde pre-kinder hasta el egreso de su educación básica. Su objetivo es mejorar el nivel de salud oral de los estudiantes adscritos a dichos módulos, mediante la recuperación y rehabilitación de las dos patologías bucodentales más prevalentes, caries y gingivitis, con especial énfasis en el área educativa, preventiva y curativa (Junta Nacional de Auxilio Escolar y Becas, 2017).
En el año 2015 el programa desarrolló 291 módulos en 278 comunas de Chile dentales, logrando una cobertura de 171.060 usuarios entre pre-kinder y $8^{\circ}$ básico de 2.896 establecimientos educacionales municipales y subvencionados. El Programa de Salud Oral cuenta desde el año 2013 con un sistema informático para el registro de antecedentes personales, clínicos, geográficos y del sistema de salud al cual pertenece, como también de otras variables: ubicación geográfica del módulo dental, dependencia de los colegios adscritos para recibir atención, previsión, su pertenencia al sistema Chile Solidario, el cual promueve la incorporación de familias y personas en situación de extrema pobreza a las redes de apoyo social, así como su acceso a mejores condiciones de vida para que superen la indigencia (Ministerio de Desarrollo Docial y Familia, 2019).

Este estudio tiene como objetivo determinar la prevalencia y severidad de caries en niños y niñas atendidos en el programa de salud oral de la JUNAEB, atendidos durante el 2015. Esta caracterización descriptiva nos permitirá identificar potenciales factores de riesgo vinculados a distribución geográfica, tipo de dependencia del establecimiento educacional, sistema de previsión y evidenciar potenciales diferencias entre niños de escasos recursos y aquellos que están en situación de extrema pobreza, que corresponden a los niños que participan del Sistema Chile Solidario. Generar esta evidencia permitirá respaldar la toma de decisiones respecto de las acciones de prevención y promoción focalizados en estos grupos vulnerables

\section{MATERIAL Y MÉTODO}

Se realizó un estudio descriptivo de corte trasversal. Los datos fueron obtenidos de las bases del Sistema Informático del Programa de Salud Oral de JUNAEB del año 2015. La base de datos, fue adaptada con el programa estadístico Stata 13 (Stata Corp LP, USA); ejecutándose procedimientos de gestión de la calidad de los datos. Las principales variables de interés fueron: presencia de lesiones de caries y la severidad medida a través del índice de ceod (dientes primarios) y índice de COPD (dientes permanentes). Las variables de asociación exploratorias fueron zona geográfica, provincia, sexo, tipo de dependencia administrativa del colegio, tipo de enseñanza, sistema de salud, situación de extrema pobreza (adscritos o no al Sistema Chile Solidario), tipo de dentición (definitiva, mixta y primaria), tipo de atención (altas de in- 
ESPINOZA-ESPINOZA, G.; PINEDA, P.; ATALA-ACEVEDO, C.; MUÑOZ-MILLÁN, P. ; MUÑOZ, S.; WEITS, A.; HERNANDEZ, B.; CASTILLO, J. \& ZAROR, C. Prevalencia y severidad de caries dental en los niños beneficiarios del programa de salud oral asociados a escuelas de Chile. Int. J. Odontostomat., 15(1):166-174, 2021.

greso, de control y atenciones de urgencia) y la distribución por zona geográfica que corresponde a una disposición propia de la JUNAEB: Zona Norte (regiones I, II, III y XV), Zona Centro (IV, V, VI y XIII), Zona Centro-sur (VII, VIII y IX), Zona Sur (X, XIV) y la Zona Austral (XI, XII). El sistema de salud se determinó según la categorización del seguro de salud estatal que poseían, el cual, para este estudio se le considera un proxi del nivel socio-económico del grupo familiar. El Fondo Nacional de Salud (FONASA) clasifica a sus beneficiarios en los siguientes tramos según su ingreso. A: Personas indigentes o carentes de recursos; $\mathrm{B}$ : Personas que perciben un ingreso imponible mensual menor o igual a CLP 250.000; C: Personas que perciben un ingreso imponible mensual mayor a CLP 250.000 y menor o igual a CLP 365.000; D: Personas que perciben un ingreso imponible mensual mayor a CLP 365.000.

Con un análisis descriptivo se determinaron las características sociodemográficas, socioeconómicas y la distribución de las variables bajo estudio. Se usaron distribuciones de frecuencia para datos nominales y ordinales; medidas de resumen para datos discretos y continuos. Para el estudio de la asociación entre variables de resultado con características biogeográficas de los estudiantes, se usaron pruebas para la comparación de promedios y pruebas para tablas de contingencia según el nivel de medición de las variables analizadas. La asociación independiente entre las variables de respuesta con las variables de exposición y de control se determinó mediante el test chi2 para la variable de respuesta dicotómica, y $t$ test en el caso de la variable de respuesta continua. En ambos casos se consideró un nivel de significación de 0,05.

Dado que el estudio contempla más de una provincia, se procedió a hacer análisis estratificados según provincia. La prevalencia de caries y de los índices de ceod y COPD por provincia fueron presentados mediante mapas de georreferenciación. Estos a través de mapas de densidad de color, muestra las zonas más y menos afectadas por la enfermedad.

\section{RESULTADOS}

La muestra quedó constituida por 162.116 individuos, siendo el $50 \%$ mujeres. La Zona Centro (53

Tabla I. Descripción de la muestra según variables basales y sexo.

\begin{tabular}{|c|c|c|c|}
\hline Población Estudio & $\begin{array}{l}\text { Femenino } \\
\mathrm{n}=81.810\end{array}$ & $\begin{array}{l}\text { Masculino } \\
\mathrm{n}=80.306\end{array}$ & $\begin{array}{l}\text { Total } \\
n=162.116\end{array}$ \\
\hline \multicolumn{4}{|l|}{ Distribución Geográfica } \\
\hline Norte & $3.060(4 \%)$ & $2.813(3 \%)$ & $5.873(4 \%)$ \\
\hline Centro & $43.281(53 \%)$ & $41.910(52 \%)$ & $85.191(53 \%)$ \\
\hline Centro Sur & $18.399(22 \%)$ & $18.509(23 \%)$ & $36.908(23 \%)$ \\
\hline Sur & $15.427(19 \%)$ & $15.435(19 \%)$ & $30.862(19 \%)$ \\
\hline Austral & $1.643(2 \%)$ & $1.643(2 \%)$ & $3.286(2 \%)$ \\
\hline \multicolumn{4}{|l|}{ Tipo de dependencia } \\
\hline Corporación Municipal & $18.176(22 \%)$ & $16.661(21 \%)$ & $34.827(21 \%)$ \\
\hline Municipal DAEM & $45.024(55 \%)$ & $45.622(57 \%)$ & $90.646(56 \%)$ \\
\hline $\begin{array}{l}\text { Particular Subvencionado } \\
\text { Tipo de enseñanza }\end{array}$ & $16.610(23 \%)$ & Tipo de enseñanza & $36.633(23 \%)$ \\
\hline Pre-escolar & $19.668(24 \%)$ & $17.934(22 \%)$ & $37.602(23 \%)$ \\
\hline \multicolumn{4}{|l|}{ Sistema de salud (475 } \\
\hline rogitsas sin información) & 76.007 (93 \%) & $74.538(93 \%)$ & $150.545(93 \%)$ \\
\hline $\begin{array}{l}\text { Isapre + otra previsión } \\
\text { Tipo de dentición (151 }\end{array}$ & $5.562(7 \%)$ & $5.534(7 \%)$ & $11.096(7 \%)$ \\
\hline Definitiva & $24.900(30 \%)$ & $23.982(30 \%)$ & $48.882(30 \%)$ \\
\hline Mixta & $39.180(48 \%)$ & $39.435(49 \%)$ & $78.615(49 \%)$ \\
\hline Primaria & $17.652(22 \%)$ & $16.816(21 \%)$ & $34.468(21 \%)$ \\
\hline \multicolumn{4}{|l|}{ Tipo de atención } \\
\hline Control & $48.513(59 \%)$ & $46.642(58 \%)$ & $95.155(59 \%)$ \\
\hline Ingreso & $33.297(41 \%)$ & $33.664(42 \%)$ & $66.961(41 \%)$ \\
\hline \multicolumn{4}{|c|}{ Situación de extrema pobreza } \\
\hline No & $56.537(69 \%)$ & $55.869(70 \%)$ & $112.406(69 \%)$ \\
\hline Si & $25.226(31 \%)$ & $24.375(30 \%)$ & $49.601(31 \%)$ \\
\hline
\end{tabular}


$\%$ de la muestra), es la que tiene mayor representación, donde la región Metropolitana aportó mayor cantidad de escolares (29\%). La distribución de las otras zonas geográficas se encuentra en la Tabla I. De los niños de esta muestra, el $56 \%$ pertenece a establecimientos municipales dependientes de la Dirección de Administración de Educación Municipal (DAEM) y el $77 \%$ corresponde a enseñanza básica. La mayor parte de la muestra (93\%) es beneficiario del seguro de salud estatal FONASA, sólo el $7 \%$ es afiliado a ISAPRES u otro sistema de salud del país. Los niños en situación de extrema pobreza adscritos al programa Chile solidario corresponden a (30,79\%). De acuerdo al tipo de dentición, $49 \%$ corresponde a escolares con dentición mixta y respecto al tipo de atención, un 59 \% correspondió a atención de control. (Tabla I).

Los resultados de prevalencia se encuentran en la Tabla II. La prevalencia de caries en la población estudiada fue de $49 \%$. La mayor prevalencia de caries se observó en la zona centro sur, con un valor de un $63 \%$. La prevalencia de caries fue muy similar en ambos sexos, sin observarse diferencias significativas. En el análisis según tipo de dependencia, se observó que los niños pertenecientes a colegios particulares subvencionados tienen un $8 \%$ menos de prevalencia de caries que los otros dos tipos de dependencia $(p<0,001)$. Al comparar la prevalencia de caries entre preescolares y escolares (49\% y $50 \%$, respectivamente), se observó que no hay diferencias $(p=0,131)$. Según el sistema de salud, los niños pertenecientes a FONASA, registraron una prevalencia de caries de un $50 \%$ comparado con los pertenecientes a las categorías "Isapre" y "otra previsión", que registraron un 43 $\%(p<0,001)$. Los niños en situación de extrema pobreza presentaron cerca de un $6 \%$ más de prevalencia de caries que los que no están en esa situación. La dentición mixta presentó la prevalencia de caries más alta $(55 \%)$, con respecto la dentición primaria $(49 \%)$ y dentición definitiva $(43 \%)(p<0,001)$. Según el tipo de atención, la prevalencia de caries en los niños que asisten a control fue de $46 \%$, observándose un $9 \%$ más bajo que los que ingresan por primera vez $(p<0,001)$.

Tabla II. Prevalencia de caries de muestra según variables sociodemográficas y clínicas

\begin{tabular}{|c|c|c|c|c|}
\hline Población Estudio & Con caries & Sin caries & Total & Valor $\mathrm{P}$ \\
\hline Variables demográficas & $81.414(49 \%)$ & $80.702(50) \%$ & $162.116(100 \%)$ & \\
\hline Norte & $2.535(41 \%)$ & $3.635(59 \%)$ & $6.170(100 \%)$ & \\
\hline Centro & $47.525(53 \%)$ & $42.376(47 \%)$ & $89.901(100 \%)$ & \\
\hline Centro Sur & $24.332(63 \%)$ & $14.445(37 \%)$ & $38.777(100 \%)$ & \\
\hline Sur & $17.611(54 \%)$ & $14.969(46 \%)$ & $32.580(100 \%)$ & \\
\hline Austral & $1.724(47 \%)$ & $1.908(53 \%)$ & $3.632(100 \%)$ & $<0,001$ \\
\hline \multicolumn{5}{|l|}{ Sexo } \\
\hline Femenino & $40.835(50 \%)$ & $49.975(50 \%)$ & $81.810(100 \%)$ & \\
\hline Masculino & $39.867(50 \%)$ & $40.439(50 \%)$ & $80.306(100 \%)$ & 0,276 \\
\hline \multicolumn{5}{|c|}{ Tipo de dependencia administrativa } \\
\hline Corporación Municipal & $18.443(53 \%)$ & $16.394(47 \%)$ & $34.837(100 \%)$ & \\
\hline Municipal DAEM & $46.351(51 \%)$ & $44.295(49)$ & $90.646(100 \%)$ & \\
\hline Particular Subvencionado & $15.908(43 \%)$ & $20.725(57) \%$ & $36.633(100 \%)$ & $<0,001$ \\
\hline \multicolumn{5}{|l|}{ Tipo de enseñanza } \\
\hline Pre-escolar & $18.590(49 \%)$ & $19.012(51 \%)$ & $37.602(100 \%)$ & \\
\hline Escolar & $62.112(50 \%)$ & $62.402(50 \%)$ & $124.514(100 \%)$ & 0,131 \\
\hline \multicolumn{5}{|l|}{ Sistema de salud } \\
\hline Fonasa & $75.705(50 \%)$ & $74.840(50 \%)$ & $150.545(100 \%)$ & \\
\hline Isapre +otra previsión & $4.737(43 \%)$ & $6.359(57 \%)$ & $11.096(100 \%)$ & $<0,001$ \\
\hline \multicolumn{5}{|l|}{ Tipo de Dentición } \\
\hline Definitiva & $20.849(43 \%)$ & $28.033(57 \%)$ & $48.882(100 \%)$ & \\
\hline Mixta & $42.996(55 \%)$ & $35.619(45 \%)$ & $78.615(100 \%)$ & \\
\hline Primaria & $16.756(49 \%)$ & $17.712(51 \%)$ & $34.468(100 \%)$ & $<0,001$ \\
\hline \multicolumn{5}{|l|}{ Tipo de Atención } \\
\hline Control & $43.627(46 \%)$ & $51.528(54 \%)$ & $95.155(100 \%)$ & \\
\hline Ingreso & $37.075(55 \%)$ & $29.886(45 \%)$ & $66.961(100 \%)$ & $<0,001$ \\
\hline \multicolumn{5}{|c|}{ Situación de extrema pobreza } \\
\hline No & $53.925(47,97 \%)$ & $58.481(52,03 \%)$ & $112.406(100 \%)$ & \\
\hline $\mathrm{Si}$ & $26.723(53,88 \%)$ & $22.878(46,12 \%)$ & $49.601(100 \%)$ & $<0,001$ \\
\hline
\end{tabular}


El promedio de índice ceod para la muestra fue de 2,48. La Zona Centro-sur presenta el promedio de caries en dientes primarios más alto $(2,98)$, siendo la Región del Bío-Bío con el promedio de caries más alto en dentición primaria $(3,51)$. Los resultados muestran diferencias significativas en el índice ceod basal según sexo, siendo los hombres los que presentan un mayor promedio $(2,54)$. En relación al tipo de dependencia administrativa se observa que el promedio ceod es más bajo en los niños de colegios particular subvencionado y en relación al tipo de enseñanza, se observó que los preescolares tienen el promedio de ceod más alto $(2,58)$ sin que la diferencia con los escolares sea significativa. Sin embargo, se registran diferencias significativas para los beneficiarios de FONASA y los niños en situación de extrema pobreza, observándose en ambas variables ceod más altos: 2,51 en los niños pertenecientes a FONASA en comparación de los afiliados a Isapres u otra previsión 2,01 y en los niños en situación de extrema pobreza muestran un valor de 2,69 comparado a 2,39 de los que no están en esa situación. El promedio de ceod es muy similar al comparar dentición mixta de dentición primaria. Por otro lado, se observó una diferencia estadísticamente significativa al comparar el índice ceod entre los escolares que acuden por primera vez $(2,61)$ comparados con los que asisten a control $(2,36)$. Los demás resultados relacionados con el índice ceod se observan en la Tabla III.

El índice COPD de la muestra es de 1,55 y al igual que para el índice ceod, la Zona Centro Sur tiene el valor más alto $(1,84)$, observándose adicionalmente que la región del Bío-Bío presenta el mayor índice COPD $(2,09)$. En el índice COPD según sexo se registra que las mujeres presentan un mayor promedio que los hombres con un 1,65 y 1,44 respectivamente lo que resulta ser una diferencia estadísticamente significativas $(p<0,001)$. Los colegios subvencionados tienen un promedio menor de COPD que los colegios municipales. De acuerdo con el sistema de salud, se observó que los escolares pertenecientes a FONASA tienen un COPD más alto $(1,57)$ en comparación de los afiliados a Isapres $u$ otra previsión, quienes presentan un promedio de caries igual a 1,17. En los niños en situación de extrema pobreza se observó un COPD más alto $(1,79)$ comparado a los no están en esa situación $(1,43)$. El promedio de COPD es mayor al comparar dentición definitiva con dentición mixta y los pacientes que acuden a control tienen un mayor promedio de índice $(1,56)$ que aquellos ingresan a tratamiento $(1,51)$. Los demás resultados relacionados con el índice COPD se observan en la Tabla III.

Las Figuras 1 a 3 muestran la tendencia epidemiológica de la caries a lo largo de Chile.

$\begin{array}{lcccc}\text { Tipo de Dentición } & & & & \\ \text { Definitiva } & - & & 2,69 & \\ \text { Mixta } & 2,48 & & 0,85 & <0,001 \\ \text { Primaria } & 2,53 & <0,001 & - & \\ \text { Tipo de Atención } & & & & \\ \text { Control } & 2,36 & & 1,56 & \\ \text { Ingreso } & 2,61 & <0,001 & 1,51 & <0,001 \\ \text { Situación de extrema pobreza } & & & & \\ \text { No } & 2,39 & & 1,43 & \\ \text { Si } & 2,69 & <0,001 & 1,79 & <0,001\end{array}$



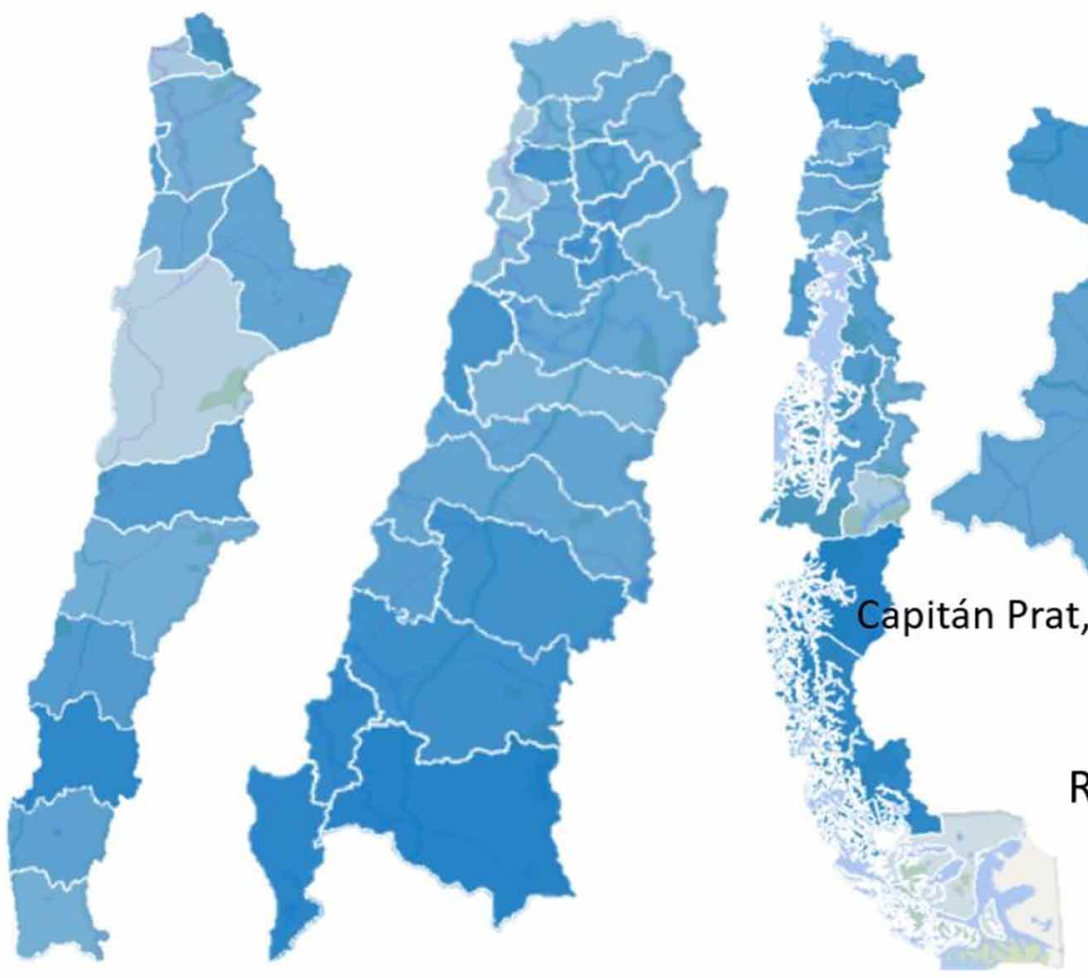

\section{Rango de prevalencia}

$0,21 \quad 0,66$

Fig. 1. Mapa de color para la prevalencia de caries según provincias de Chile, Programa JUNAEB, Chile, 2015.
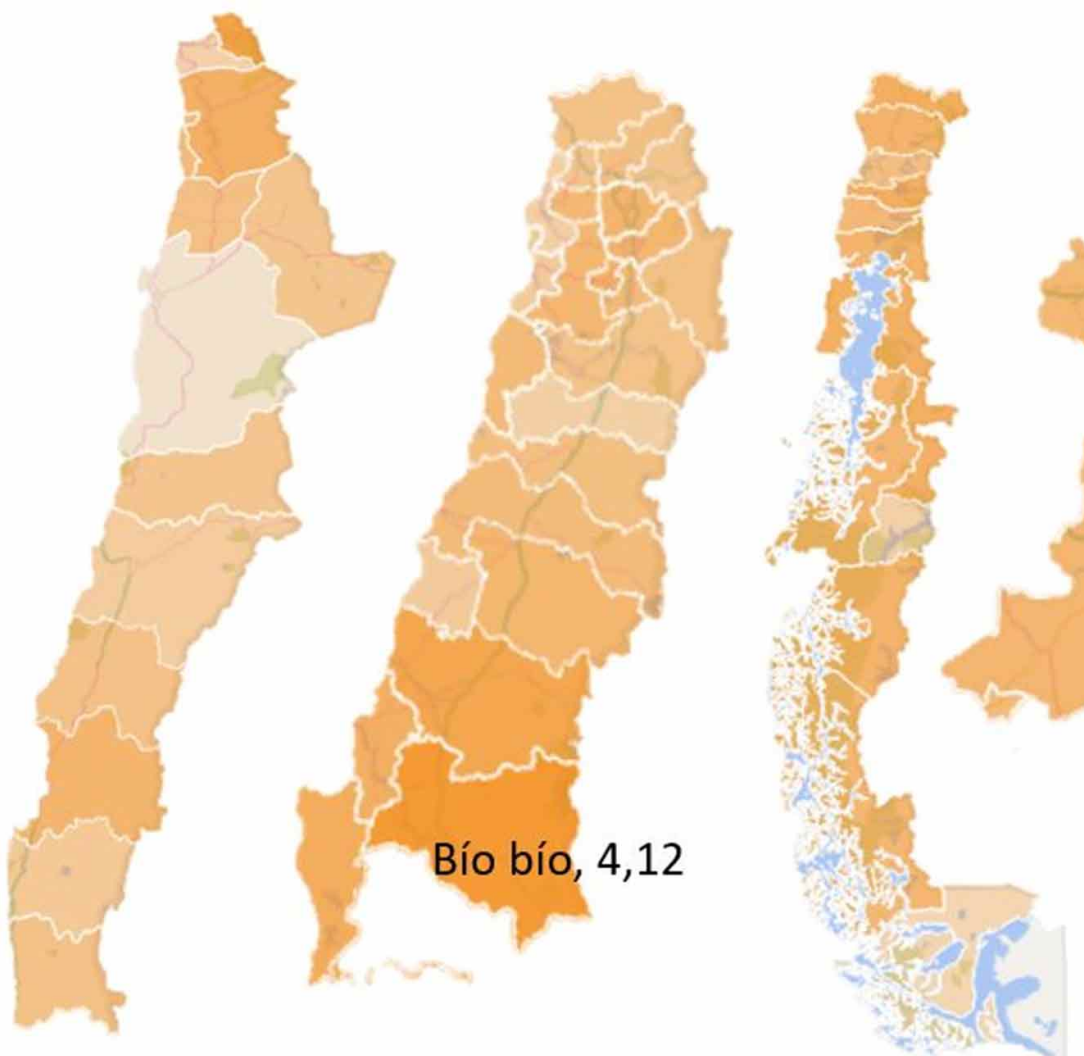

Fig. 2. Mapa de color para los valores del incide CEOD según provincias de Chile, Programa JUNAEB, Chile, 2015. 

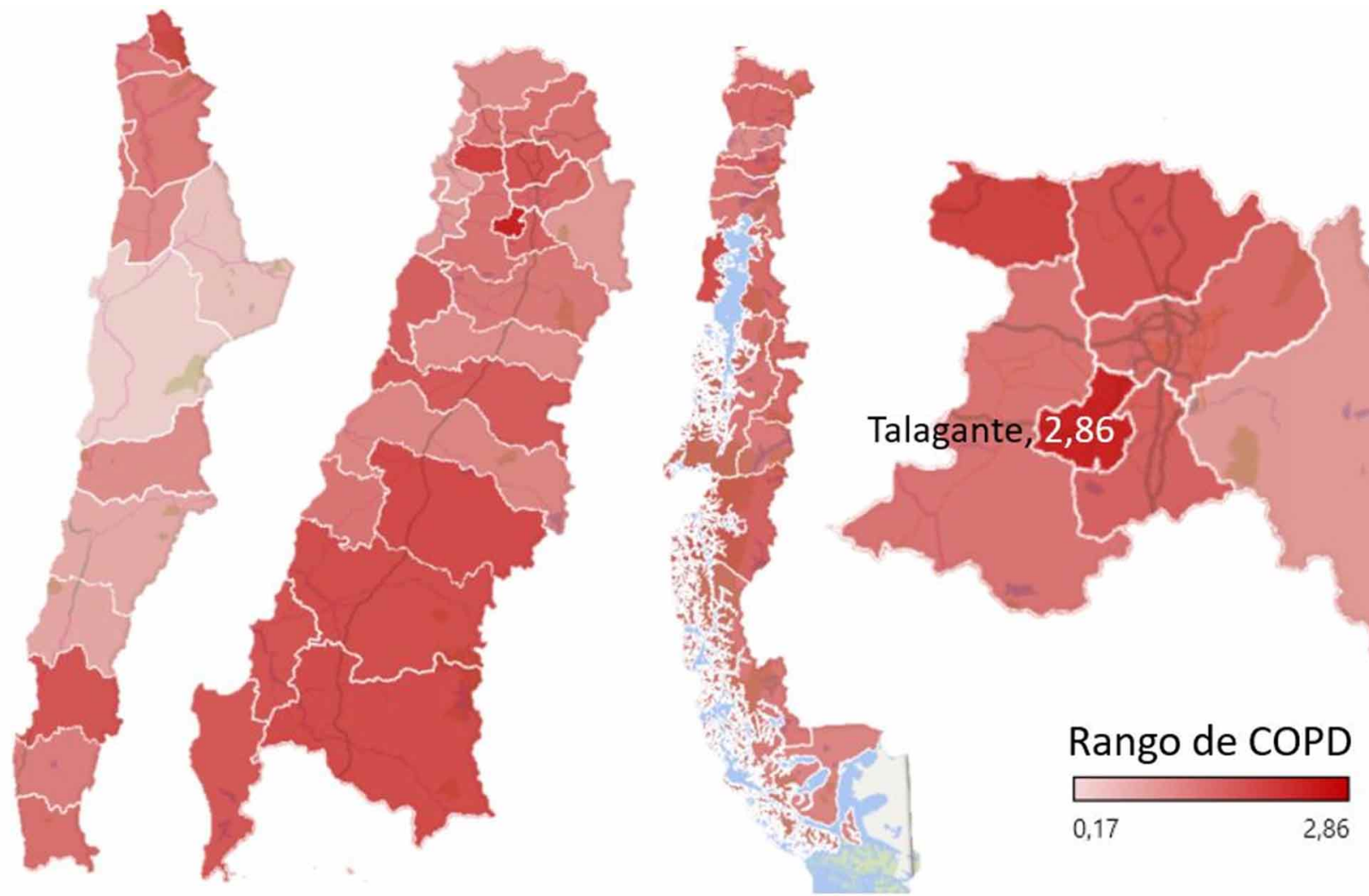

Fig. 3 Mapa de color para los valores del incide COPD según provincias de Chile, Programa JUNAEB, Chile, 2015.

\section{DISCUSIÓN}

El Programa de Salud Oral de la Junta Nacional de Auxilio Escolar y Becas (JUNAEB) de Chile, atiende niños desde Pre-Kinder a 8avo básico (aproximadamente desde 4 a 16 años de edad). Esta población asignada, que recibe atención dental asociada a escuelas y que en su mayoría corresponden a niños de escasos recursos, tiene una prevalencia de casi la mitad de la población (49\%). En Chile no existen datos de la prevalencia general de la población en este amplio rango de edad, limitándose sólo en grupos etarios específicos. Al comparar nuestros resultados con los datos existentes del Ministerio de Salud del Gobierno de Chile (MINSAL), se observa que la prevalencia es menor, dado que los indicadores ministeriales muestran una prevalencia de caries a los 6 años de $70,4 \%$ y a los 12 años de $62,5 \%$. En relación a otros estudios epidemiológicos a nivel nacional, este estudio también muestra un valor de prevalencia menor a los reportes de Fernández González et al. y Gaete Forno et al. (2014). También podemos observar que la prevalencia en este estudio es menor, que lo repor- tado por la Organización Mundial de la Salud (OMS) que identifica la caries como una de las enfermedades crónicas más importantes del mundo, afectando entre el 60 al $95 \%$ de los niños de países desarrollados y en vias de desarrollo, con tasas especialmente altas en América Latina y Asia (World Health Organization, 2013). Por su parte, Kassebaum et al. (2015) reporta que 2.400 millones de personas en dentición permanente y 621 millones de niños en dentición primaria presentan caries no tratadas alrededor del mundo. Si bien se trata de una población más vulnerable y de mayor riesgo de caries, es importante considerar como lo reporta Fuentes et al. (2014), que es una población que recibe tratamiento integral sistemáticamente durante su periodo escolar, con una estrategia de educación en salud oral bastante estructurada. Esto podría justificar que la prevalencia sea de menor que los reportes anteriores y que el reporte mundial sobre carga de enfermedad de caries (Kassebaum et al.). Éste último, concluye que la caries en los dientes primarios fue la décima enfermedad más prevalente, siendo la edad más afectada la de 6 años, con una prevalencia del $85 \%$ para los países del sur de América Latina. 
ESPINOZA-ESPINOZA, G.; PINEDA, P.; ATALA-ACEVEDO, C.; MUÑOZ-MILLÁN, P. ; MUÑOZ, S.; WEITS, A.; HERNANDEZ, B.; CASTILLO, J. \& ZAROR, C. Prevalencia y severidad de caries dental en los niños beneficiarios del programa de salud oral asociados a escuelas de Chile. Int. J. Odontostomat., 15(1):166-174, 2021.

La severidad de la caries dental, medida a través de los índices de ceod y COPD fue de 2,48 y 1,55 respectivamente. Como este es un promedio que incluye niños de un amplio espectro de edades, es difícil comparar con el perfil epidemiológico del país que entrega valores de edades muy específicas, como un ceod de 3,71 a los 6 años y un COPD de 1,9 (Soto et al.).

Nuestros resultados mostraron que la prevalencia de caries fue muy similar en ambos sexos, sin observarse diferencias significativas, coincidiendo con los resultados publicados por Hoffmeister et al. (2016) y Fernández González et al.

Otras observaciones son, que los índices de ceod y COPD son más altos en los niños que pertenecen a FONASA comparados a Isapre u otra previsión y que los niños pertenecientes a colegios particulares subvencionados tienen significativamente una menor prevalencia de caries que los otros dos tipos de dependencia ( $8 \%$ menos). También los niños en situación de extrema pobreza registran valores mayores. Estos indicadores son coherentes con la literatura en donde las variables económicas, tales como la previsión, la dependencia del colegio, y la situación de extrema pobreza, muestran asociación positiva con la prevalencia y severidad de la caries (dos Santos Junior et al., 2014; Baggio et al., 2015).

Los mapas de georreferencia muestran que existen fuertes diferencias en los indicadores de salud oral según provincia. La mayor prevalencia se registra en la provincia de Capitán Prat, que presenta difíciles condiciones de acceso a la atención dental. EI COPD más alto se observó en la provincia de Talagante, que tienen altos índices de ruralidad y el ceod más alto se observó en la provincia del Bio-bio, que presenta niveles bajos de agua fluorada $(0,003$ a $0,055 \mathrm{ppm}$ ), lo que muestra cierta concordancia con el reporte de Espinoza-Espinoza et al. (2016) que señala un ceod alto $(2,53)$ en zona rurales no fluoradas de la región La Araucanía.

Una limitación de este estudio es que no considera las caries no cavitadas, lo cual podría subestimar los resultados obtenidos, sin embargo, lo hace comparable con otros estudios epidemiológicos, dado que cumple con lo establecido por la OMS (los 12 años como la edad global de vigilancia de la caries en comparaciones internacionales y de vigilancia de tendencias de la enfermedad). Por otra parte, la validez externa está afectada, porque incluye a los preescola- res y escolares que asisten al programa JUNAEB, sin embargo, dado la amplia distribución del programa y que los datos pertenecen a casi la totalidad del universo de los pacientes, se espera que los valores obtenidos sean muy representativos de la población atendida por la JUNAEB que corresponde a niños de escasos recursos de Chile. Otra limitación es la existencia de múltiples observadores, efecto que tiende a diluirse por alto número de registros incluidos. La atención dental integral asociada a los colegios, estrategia otorgada por el equipo de salud oral de la JUNAEB, para brindar atención dental a población vulnerable a lo largo de todo Chile, es una de las intervenciones en salud oral más completas en el país, que junto al programa GES de 6 años, han contribuido a cumplir lo establecido por los Objetivos Sanitarios de Salud, 2000-2010 disminuir la caries y aumentar el acceso a la atención odontológica a un 50 \%, en población menor de 20 años.

En conclusión, este estudio aporta evidencia sobre la asociación de la caries y el nivel socioeconómico, ya que todas las variables económicas, tales como la previsión, la dependencia del colegio, y la situación de extrema pobreza, muestran asociación positiva con la prevalencia y severidad de la caries. Por último, la distribución geográfica de la caries permite identificar zonas geográficas donde es necesario estudiar medidas preventivas que permitan compensar la ruralidad o la falta de flúor en el agua.

ESPINOZA-ESPINOZA, G.; PINEDA, P.; ATALAACEVEDO, C.; MUÑOZ-MILLÁN, P. ; MUÑOZ, S.; WEITS, A.; HERNANDEZ, B.; CASTILLO, J. \& ZAROR, C. Prevalence and severity of dental caries in beneficiary children in the oral health program associated with schools in Chile. Int. J. Odontostomat., 15(1):166-174, 2021.

ABSTRACT:Caries is the most prevalent chronic disease in children, constituting a worldwide public health problem. The aim of this study was to determine the prevalence and severity of caries in children included in the Oral Health Program associated to schools of the National Board of School Aid and Scholarships (JUNAEB). A crosssectional study based on data from 2015 electronic register JUNAEB Oral Health Program was carried out. The main variables studied were presence and severity of caries (dmft and DMFT indices) and association variables were geographical area, sex, type of administrative dependency of the school, type of education, health system, and situation of extreme poverty, type of teething and type of care. The independent association between the variables was analyzed using the Chi2 test and the t-test.The sample consisted of 162,116 individuals, $50 \%$ being women. The studied 
population showed a prevalence of $49 \%$ and a CEOD and COPD index of 2.48 and 1.55 , respectively. The highest prevalence $(63 \%)$ was the south-central zone and the BíoBío region showed the highest severity indices $(p<0.001)$. The most significant associations were between caries and socioeconomic level and geographic area $(p<0.001)$. This study shows the association between caries prevalence / severity and socioeconomic level, and the geographical distribution of caries, which make necessary the implementation of preventive measures that compensate rurality, or the lack of water fluoridation in some areas of extreme poverty.

KEY WORDS: dental caries, risk factors, child, prevalence, socioeconomic factors.

\section{REFERENCIAS BIBLIOGRÁFICAS}

Baggio, S.; Abarca, M.; Bodenmann, P.; Gehri, M. \& Madrid, C. Early childhood caries in Switzerland: a marker of social inequalities. BMC Oral Health, 5(1):1-9, 2015

Cabrera, C.; Arancet, M. I.; Martínez, D.; Cueto, A. \& Espinoza, S. Oral health in urban and rural school population. Int. J. Odontostomat., 9(3):341-8, 2015.

Cerón-Bastidas, X. El sistema ICDAS como método complementario para el diagnóstico de caries dental. Rev. CES Odontol., 28(2):1009, 2015.

Corrêa-Faria, P.; Paixão-Gonçalves, S.; Paiva, S. M. \& Pordeus, I. A. Incidence of dental caries in primary dentition and risk factors: a longitudinal study. Braz. Oral Res., 30(1):e59, 2016.

dos Santos Junior, V. E.; de Sousa, R. M. B.; Oliveira, M. C.; de Caldas Junior, A. F. \& Rosenblatt, A. Early childhood caries and its relationship with perinatal, socioeconomic and nutritional risks: a cross-sectional study. BMC Oral Health, 14:47, 2014.

Espinoza-Espinoza, G.; Muñoz-Millán, P.; Vergara-González, C.; Atala-Acevedo, C. \& Zaror, C. Prevalence of early childhood caries in non-fluoridated rural areas of Chile. J. Oral Res., 5(8):307-13, 2016.

Fernández González, C.; Núñez Franz, L. \& Díaz Sanzana, N. Determinantes de salud oral en población de 12 años. Rev. Clin. Periodoncia Implantol. Rehabil. Oral., 4(3):117-21, 2011.

Fuentes, N. J.; Corsini, M. G.; Bornhardt, T.; Ponce, V. A. \& Ruiz, F. Á. Prevalence of dental caries and oral hygiene level in children aged 6 years attended under GES and JUNAEB Models. Int. J. Odontostomat., 8(3):385-91, 2014.

Gaete Forno, M. J.; Córdova Cisterna, C. \& Oliva Mella, P. Oral health status and dental assistance control in 12-year-old school children in the Penco Municipality, Biobío Region. Int. J. Odontostomat., 7(3):389-94, 2013.

Giacaman, R. A.; Bustos, I. P.; Bravo-León, V. \& Mariño, R. J. Impact of rurality on the oral health status of 6-year-old children from central Chile: the EpiMaule study. Rural Remote Health, 15(2):3135, 2015.

Hernández-Vásquez, A.; Azañedo, D.; Díaz-Seijas, D.; BendezúQuispe, G.; Arroyo-Hernández; H.; Vilcarromero, S. \& AgudeloSuárez, A. A. Acceso a servicios de salud dental en menores de doce años en Perú, 2014. Salud Colect., 12(3):429-41, 2016.

Hoffmeister, L.; Moya, P.; Vidal, C. \& Benadof, D. Factors associated with early childhood caries in Chile. Gac. Sanit., 30(1):59-62, 2016.

Junta Nacional de Auxilio Escolar y Becas (JUNAEB). Manual de Procedimientos de Auditorías Odontológicas del Departamento de Salud del Estudiante. Santiago de Chile, Gobierno de Chile, Jun- ta Nacional de Auxilio Escolar y Becas, 2017. Disponible en: http:/ /anfitrion.cl/GobiernoTransparente/junaeb/2018/otras/153.pdf

Kassebaum, N. J.; Bernabé, E.; Dahiya, M.; Bhandari, B.; Murray, C. J. L. \& Marcenes, W. Global burden of untreated caries: a systematic review and metaregression. J. Dent Res., 94(5):650$8,2015$.

Lambert, M. J.; Vanobbergen, J. S. N.; Martens, L. C. \& De Visschere, L. M. J. Socioeconomic inequalities in caries experience, care level and dental attendance in primary school children in Belgium: a cross-sectional survey. BMJ Open, 7(7):e015042, 2017.

Matamala-Santander, A.; Rivera-Mendoza, F. \& Zaror, C. Impact of caries on oral health related quality of life in adolescents: a systematic review and meta-analysis. Int. J. Odontostomat., 13(2):219-29, 2019.

Ministerio de Desarrollo Docial y Familia (MDSF). Chile Solidario. Descripción, 2019. Santiago de Chile, Ministerio de Salud, Gobierno de Chile, 2019. Disponible en: http://www.ips.gob.cl/servlet/ internet/content/1421810829144/chile-solidario

Ministerio de Salud (MINSAL). Encuesta Nacional de Salud ENS Chile 2009-2010. Santiago de Chile, Ministerio de Salud, Gobierno de Chile, 2011b. Disponible en: https://www.minsal.cl/portal/url/item/ bcb03d7bc28b64dfe040010165012d23.pdf

Ministerio de Salud (MINSAL). Informe Consolidado del Diagnóstico Nacional de Salud bucal de los Niños y Niñas de 2 y 4 Años que Participen en Educación Parvularia. Chile 2007-2010. Santiago de Chile, Ministerio de Salud, Gobierno de Chile, 2011a. Disponible en: https://diprece.minsal.cl/wrdprss_minsal/wp-content/ uploads/2015/05/Informe-consolidado-2-y-4-a \%C3 \%B1os.pdf

Ministerio de Salud de Chile (MINSAL). Para la ejecución del programa Sembrando Sonrisas, 2018. Santiago de Chile, Ministerio de Salud, Gobierno de Chile, 2018. Disponible en: https:// diprece.minsal.cl/wrdprss minsal/wp-content/uploads/2018/05/ Orientación-Técnica-Programa-Sembrando-Sonrisas-2018.pdf

Ortiz, A. S.; Tomazoni, F.; Knorst, J. K. \& Ardenghi, T. M. Influence of socioeconomic inequalities on levels of dental caries in adolescents: A cohort study. Int. J. Paediatr. Dent., 30(1):42-9, 2020.

Palomer Roggerone, L. Inequidades en salud bucal. Factores que determinan su realidad en Chile. Acta Bioeth., 22(2):315-9, 2016.

Soto, L. \& Tapia, R. Diagnóstico Nacional de Salud Bucal del Adolescente de 12 años y Evaluación del Grado de Cumplimiento de los Objetivos Sanitarios de Salud Bucal 2000-2010, 2007. Santiago de Chile, Gobierno de Chile, Ministerio de Salud, 2007. Disponible en: https://www.minsal.cl/portal/url/item/ 7f2e0f67ebbc1bc0e04001011e016f58.pdf

Soto, L.; Tapia, R.; Jara, G. \& Rodríguez, G. Diagnóstico Nacional de Salud Bucal del Niño de 6 Años. Santiago de Chile, Gobierno de Chile, Ministerio de Salud, 2007. Disponible en: https:// diprece.minsal.cl/wrdprss_minsal/wpcontent/uploads/2015/05/ Diagnostico-Nac-Salud-Bucal-ni \%C3 \%B1os-6-a \%C3 \%B1os Chile-2007.pdf

World Health Organization (WHO). Oral Health Surveys: Basic Methods. 5th ed. Ginebra, World Health Organization, 2013.

Dirección para correspondencia

A/Prof. Carlos Zaror

Manuel Montt \#112

Facultad de Odontología

Universidad de la Frontera

Temuco

CHILE

E-mail: carlos.zaror@ufrontera.cl 